\title{
Australian Funnel-Web Spider Venom Analyzed With On-Line RP-HPLC Techniques
}

\section{David Wilson and Paul Alewood}

\section{Introduction}

Venoms have attracted significant study in recent years as a reservoir of complex libraries of natural products possessing a wide range of biological activities. Moreover, venoms contain specific and potent molecules that may be utilized in pharmaceutical development and in the production of environmentally friendly insecticides. The compositions of venoms are typically highly complex and contain a variety of molecules including proteins, peptides, and numerous types of small molecules. This complexity requires highly sensitive techniques to allow separation of these components for study. The techniques should also be able to accommodate large variations in sample size to account for the differences in venom available from different creatures (e.g., some snakes can supply up to $500 \mathrm{mg}$ of crude venom from a single milking, whereas some small insects, such as ants, supply submicrogram amounts [1]). These qualities have been found and continue to be advanced in the technique highperformance liquid chromatography (HPLC), in particular reversed-phase HPLC (RP-HPLC). This technique combined with a variety of detection methods can allow the collection of a significant amount of data from very small venom samples.

Early analytical studies of whole venom samples used techniques such as gel electrophoresis, size exclusion, and ion exchange chromatography $(2,3)$. Although these techniques are still useful today, they provide limited information. Gel electrophoresis provides an idea of the overall size distribution of the protein components of the venom that typically have masses greater than $15 \mathrm{kDa}$. However, gel electrophoresis is limited in the separation it can provide 
for closely related homologs and components containing noncharged posttranslational modifications. Furthermore, the amount of sample required for gel electrophoresis is typically larger than that required for HPLC analysis (at least 5-10-fold; see Note 1) and recollection of separated components for further analysis is relatively labor intensive. Perhaps the greatest limitation of this technique is the poor accuracy of molecular weight estimation. Masses are estimated from a standard curve generated from a molecular weight marker ladder run alongside the sample and are often very inaccurate. By comparison, size exclusion chromatography has an advantage that it may be performed using small columns, though the inability to provide satisfactory component separation remains.

More recently, advances in HPLC support the high resolution separation of complex mixtures and, thus, provide a desirable technique for venom analysis. Current HPLC systems, in contrast to the early systems, provide great separation of very small quantities of complex mixtures at low flow rates, resulting in improved sample recovery.

\subsection{Liquid Chromatography-Mass Spectrometry as a Venom Fingerprinting Technique}

The detectors typically used with HPLC are nondestructive UV or diode array, but mass spectrometers can also be used and this technique is referred to as liquid chromatography-mass spectrometry (LC-MS). In fact, if set up correctly, both UV and MS data can be collected from a single run (see also Chapter 9).

Because a majority of venom toxins are peptidic or proteinaceous in character, there is a direct link between the toxin component expressed and the genetic coding of the particular specimen. The genetic coding of a group of individual specimens belonging to a particular species should have identical or very similar physicochemical properties with each toxin (peptide/protein) having a defined protein sequence, molecular weight and chromatographic retention time in RP-HPLC analyses. Therefore, the collection and comparison of this data provides a means of establishing a venom component profile for a particular species. The usefulness of this approach of identification through venom profile analysis is illustrated in many recent RP-HPLC/matrix-assisted laser desorption ionization time-of-flight mass spectrometry (RP-HPLC/MALDI-TOF) (see Note 2) examples including bacteria (4-8), snake venom (9), cone snail venom (10-12), scorpion venom (13), and tarantula venom studies (14-16).

The Australian Funnel-web spiders are a group of venomous arachnids comprising more than 35 species from two genera (Atrax and Hadronyche), which are located predominantly along the southeast coast of Australia. The current taxonomy of the Australian Funnel-web spiders is shrouded in confusion and, 

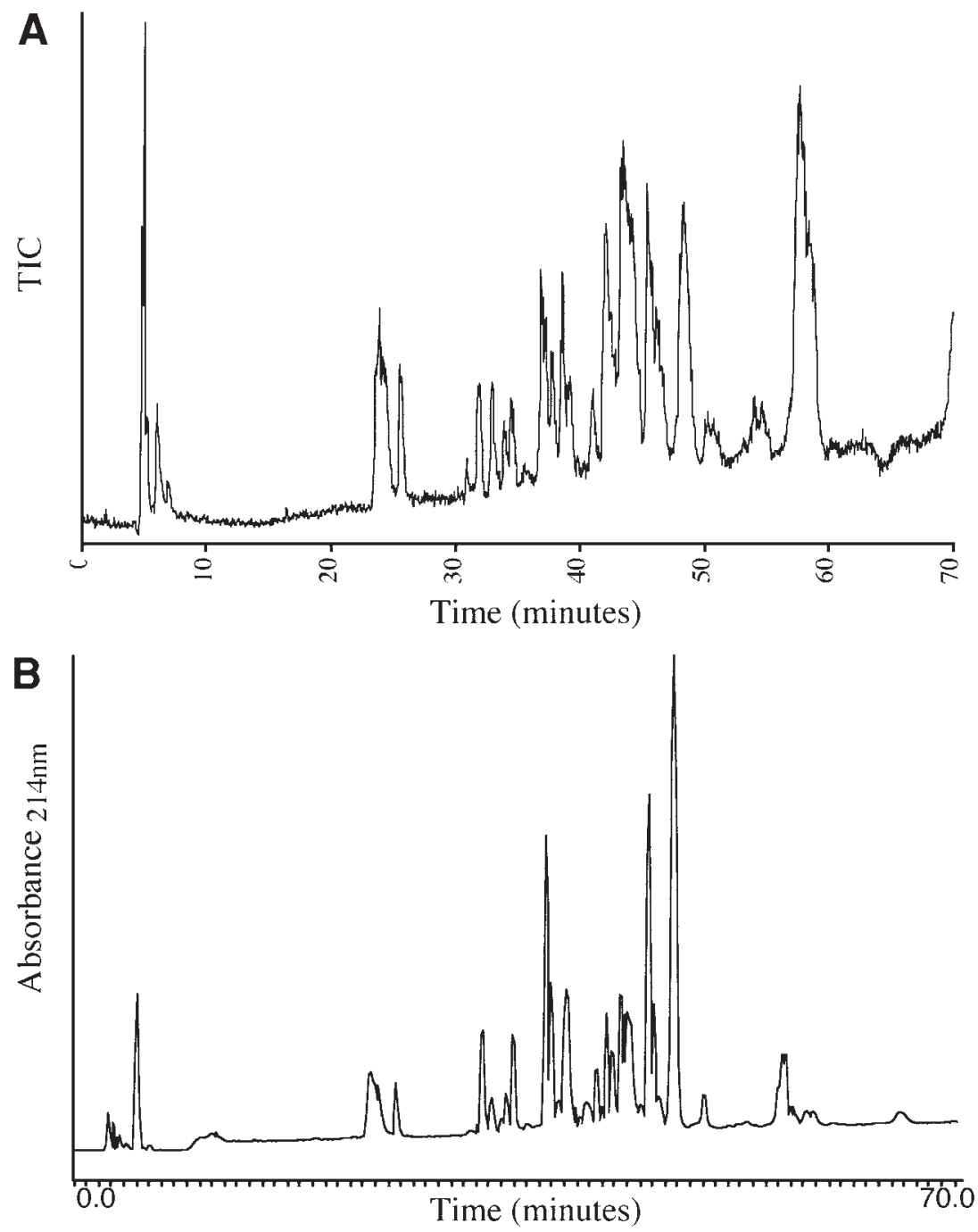

Fig. 1. (A) RP-HPLC/ESI-MS chromatogram. (B) RP-HPLC/UV chromatogram of venom from an Australian Funnel-web spider illustrating the number of components and complexity.

as a result, species identification based on morphology can prove challenging. The venom of the Australian Funnel-web spiders is complex, containing between 40 and 100 peptide components (see Fig. 1). Furthermore, the composition of the venom from certain species of these spiders has been shown to differ between the male and female specimens (17). In this chapter, we investigate the peptidic venoms of selected Australian Funnel-web spiders using 
RP-HPLC techniques, including RP-HPLC/electrospray ionization mass spectrometry (ESI-MS) (see Note 3), in order to identify their complexity, the types of molecules present and the extent to which this approach can identify and characterize different species of the Australian Funnel-web spiders.

\section{Materials}

\subsection{Venom Collection}

1. Polyethylene/polypropylene pipet tips and collection tubes (e.g., $1.5-2 \mathrm{~mL}$ Eppendorf) (see Note 4).

2. 10-100 $\mu$ L Pipet with tip ejection arm (e.g., Gilson P100).

\subsection{RP-HPLC/ESI-MS}

1. HPLC grade acetonitrile and trifluoroacetic acid (TFA).

2. Solvent A: $0.1 \%$ TFA in water.

3. Solvent B: $0.09 \%$ TFA in $90 \%$ acetonitrile $/ 10 \% \mathrm{H}_{2} \mathrm{O}$.

4. $\mathrm{RP}_{18}$ analytical column (Vydac; $2.1 \times 250 \mathrm{~mm}, 5 \mu \mathrm{m}$ particle size, $300 \AA$ pore size) (see Note 5).

5. Hewlett Packard Series1100 HPLC pump system (Hewlett Packard, Australia) or similar low flow-rate HPLC pump system such as Applied Biosystems 140B solvent delivery system.

6. Electrospray ionisation mass spectrometer with turbospray facility (e.g., PE-SCIEX triple quadrupole mass spectrometer (PE-SCIEX, Canada) equipped with an Ionspray atmospheric pressure ionization source and Turbospray interface) (see Notes 1, 2 and $\mathbf{3}$ ).

7. Applied Biosystems $785 \mathrm{~A}$ programmable absorbance detector.

\subsection{RP-HPLC}

1. HPLC grade acetonitrile and TFA.

2. Solvent A: $0.1 \%$ TFA in water.

3. Solvent B: $0.09 \%$ TFA in $90 \%$ acetonitrile $/ 10 \% \mathrm{H}_{2} \mathrm{O}$.

4. Polyethylene/polypropylene pipet tips and collection tubes $(14 \mathrm{~mL}$ Falcon or $1.5-2 \mathrm{~mL}$ Eppendorf).

5. Waters 600 HPLC controller.

6. Waters 486 tunable absorbance detector.

7. $\mathrm{RP}_{18}$ analytical column (Vydac; $4.6 \times 250 \mathrm{~mm}, 5 \mu \mathrm{m}$ particle size, $300 \AA$ pore size).

\section{Methods}

\subsection{Venom Collection}

1. Collect the venom from live specimens (see Note 6). Funnel-web spider venom is "milked" from the tips of the fangs of an aggravated specimen using a pipet equipped with a polyethylene/polypropylene tip. Collect, handle and store the venom using polyethylene/polypropylene materials (e.g., pipet tips, Eppendorf 
tubes, and so on) (see Note 7). Wash the "milked" venom from the pipet tip with $800 \mu \mathrm{L}$ of $0.1 \% \mathrm{TFA} / \mathrm{H}_{2} \mathrm{O}$ and lyophilize in a Savant Speedvac (see Note 8).

\subsection{Online RP-HPLC/ESI-MS}

1. Reconstitute the lyophilized venom samples to a concentration of approx $1 \mathrm{mg} / \mathrm{mL}$ in approx 30\% acetonitrile/water (v/v) (see Note 9).

2. Spin the venom samples in a bench centrifuge for approx $30 \mathrm{~s}$ at maximum speed to pellet all insoluble and particulate material (dirt, cell membrane in the case of dissections) (see Note 10).

3. Set up the mass spectrometer (i.e., turn mass spectrometer on and set data collection parameters) and turbospray to warm up.

4. Equilibrate the RP-HPLC column by running $100 \%$ solvent A through at the flow rate to be used for 20-30 min, but without the column eluent attached to the mass spectrometer (see Note 11). For the final 5-10 min of equilibration, attach the output of the RP-HPLC column to the mass spectrometer and monitor the mass spectrometer signal to make sure the signal is clean and free of contaminants (see Note 12).

5. Inject samples $(10 \mu \mathrm{L})$ onto an on-line $\mathrm{RP} \mathrm{C}_{18}$ analytical column and simultaneously start both the chromatography gradient and mass spectrometer data collection (see Note 13). Elute the venom constituents with a flow rate of $130 \mu \mathrm{L} / \mathrm{min}$ using a linear gradient of $100 \%$ solvent A: $0 \%$ solvent B to $60 \%$ solvent B over $60 \mathrm{~min}$. The data analysis and subsequent processing is performed using the Biomultiview (PE-SCIEX, Canada) software package. Electrospray mass spectra are acquired in positive ion mode by direct flow of the column eluent into a PE-SCIEX API III triple quadrupole mass spectrometer (PE-SCIEX, Canada) equipped with an Ionspray atmospheric pressure ionization source and Turbospray interface. The full scan data for each venom sample is acquired at an orifice potential of $80 \mathrm{~V}$ over a mass range of 400-2100 Daltons with a step size of 0.2 Dalton (see Note 14).

\subsection{Analytical RP-HPLC}

1. Dissolve lyophilized crude venom in $0.1 \% \mathrm{TFA} / \mathrm{H}_{2} \mathrm{O}$ to a concentration of approx $1 \mathrm{mg} / \mathrm{mL}$.

2. Load the venom using a $10-20 \mu \mathrm{L}$ injection from a Waters 717 Autosampler onto $\mathrm{a} \mathrm{C}_{18}$ analytical RP-HPLC column (Vydac $4.6 \times 250 \mathrm{~mm}, 300 \AA$ pore size, $5 \mu \mathrm{m}$ particle size).

3. Elute the venom components with a linear $1 \%$ gradient from 0 to $80 \%$ Solvent B over $80 \mathrm{~min}$ at a flow rate of $1 \mathrm{~mL} / \mathrm{min}$ governed by a Waters 600 Controller. Monitor the UV absorbance at $214 \mathrm{~nm}$ using a Waters 486 tunable absorbance detector.

\subsubsection{Venom Profiling of Australian Funnel-Web Spider Venom}

The RP-HPLC/UV and RP-HPLC/ESI analysis of venom from juvenile male, mature female, and mature male specimens from two species of the Atrax genus of Australian Funnel-web spiders illustrates the complexity of these venoms, the gender differences evident in some species, and how the venom 

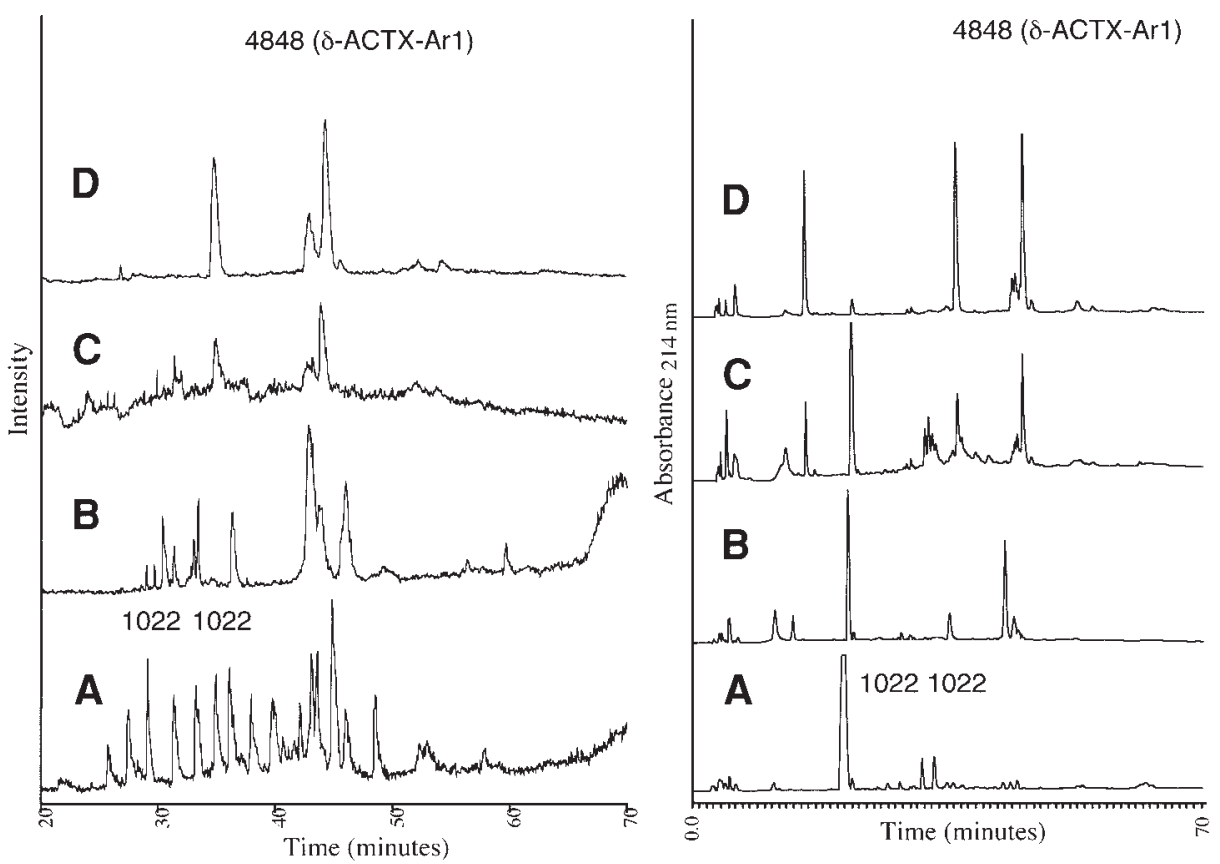

Fig. 2. A comparison of the RP-HPLC/ESI-MS and RP-HPLC/UV chromatograms from the female, and juvenile and mature male venom of Atrax robustus: Sydney. RP-HPLC/ESI-MS (left panel) and RP-HPLC/UV (right panel) chromatogram data obtained for the (A) female Atrax robustus: Sydney venom sample. (B) Mature male Atrax robustus: Sydney venom sample. (C) Prematuring moult of juvenile male Atrax robustus: Sydney venom sample. (D) Postmaturing moult mature male Atrax robustus: Sydney venom sample. Components of note are highlighted.

profile can be used as a taxonomic tool. Furthermore, the study illustrates the importance of both RP-HPLC/UV and RP-HPLC/ESI data when establishing the venom profile.

\subsubsection{RP-HPlC-Based Venom Mass Profile Analysis of THE Sydney Atrax ROBUStUS: Sydney SPECIMENS}

The venom of the Sydney Funnel-web spider (Atrax robustus: Sydney) has been extensively studied and thus was used primarily as a "control" to establish the viability of the methodology developed in these studies. As a result of the commonly known gender difference between the venom composition of this species (17), venom samples of juvenile male, mature female, and mature male specimens were investigated. The analysis of the venom samples by RP-HPLC/UV and RP-HPLC/ESI (see Fig. 2) showed a number of obvious 
differences between the chromatograms, and further confirmed the genderrelated venom composition differences. Closer examination of the mass data combined with the elution time data (see Table 1) revealed a number of components common to both genders, but also further highlighted the differences in venom composition. The most obvious difference is the dominating presence of the molecule $\delta$-ACTX-Ar1 eluting at approx $42 \mathrm{~min}$ in the RP-HPLC/UV and RP-HPLC/ESI chromatograms of the male specimen venom samples. In the juvenile male venom sample this component is evident, but at lower levels. Likewise, the female venom sample does not contain the molecule $\delta$-ACTX-Ar1, but possesses two components with $M r=1022$ Daltons not observed in the male venom profile. Several other differences are also evident upon examination of the mass data and are illustrated in Table 1. The obvious differences between the corresponding peak intensities of the RP-HPLC/UV and RP-HPLC/ESI-MS chromatograms illustrate the importance of data collection from both detectors. As the intensity of the component peaks in the RP-HPLC/ESI-MS chromatogram are related to the component's capacity to ionize in the mass spectrometer, it does not provide a adequate representation of the relative abundances of each component. In contrast, the RP-HPLC/UV data are directly related to the component concentration and, thus, provide a better profile of the relative abundances of the components in the venom profile. Furthermore, the RP-HPLC/UV data provide evidence of molecules not detected by the mass spectrometer owing to masses outside the scanned mass range or their inability to ionize (see Note 15).

\subsubsection{RP-HPlC-Based Venom Mass Profile Analysis of the AtraX SP.: ILlaWARRa SPECIMENS}

Analysis of the venom of a second species of Atrax Funnel-web spider (Atrax $s p .:$ Illawarra) provided an excellent example of the differences in venom composition observed between specimens belonging to a distinct species. RP-HPLC/UV and RP-HPLC/ESI analysis of the venom of mature male, mature female, and juvenile male (which matured in captivity and provided pre- and postmaturing moult samples) specimens revealed a very different venom composition to the Atrax robustus: Sydney specimens, and possessed some degree of gender-related venom composition differences.

The RP-HPLC/ESI-MS analysis (see Fig. 3) revealed that the venom composition of the male and female Atrax sp.: Illawarra specimens is very similar. Close inspection of the RP-HPLC/ESI-MS mass data revealed some subtle gender-related differences such as the presence of the molecules $\mathrm{Mr}=5666$ Daltons, $M r=3820$ Daltons, $M r=4519$ Daltons, $M r=4911$ Daltons, and $M r=4181$ Daltons in the venom of the male specimens only (see Table 2). Likewise, the components $M r=4410$ Daltons and $M r=4361$ Daltons were only evident in the venom of the female specimens (see Table 2). The RP-HPLC/UV 
Table 1

\section{Venom Mass Profile "Descriptor"a Molecules Determined for Male and Female Specimens of Atrax robustus: Sydney}

\begin{tabular}{|c|c|c|}
\hline $\begin{array}{l}\text { Elution time } \\
(\mathrm{min})\end{array}$ & $\begin{array}{l}\text { Female components } \\
\qquad(M r=\mathrm{Da})\end{array}$ & $\begin{array}{l}\text { Male components } \\
\qquad(M r=\mathrm{Da})\end{array}$ \\
\hline Approx 26 & 4004 & 4004 \\
\hline Approx 28 & 4395 & \\
\hline Approx 29 & 3879 & 3879 \\
\hline Approx 2.5 & 4379 & 4379 \\
\hline Approx 31 & 1022 & \\
\hline Approx 33 & 1022 & \\
\hline Approx 34 & & 7155 \\
\hline Approx 35 & 7139 & 7139 \\
\hline Approx 36 & 4615 & \\
\hline Approx 38.5 & 4498 & \\
\hline Approx 40 & 4469 & \\
\hline Approx 40.5 & 7946 & \\
\hline Approx 41 & 4811 & \\
\hline Approx 41.5 & 7915 & \\
\hline Approx 43 & 4120 & 4848 \\
\hline Approx 43.5 & 7136 & 7138 \\
\hline Approx 44 & 7286 & 7287 \\
\hline Approx 44.5 & 4547 & 7235 \\
\hline Approx 45 & 4223 & \\
\hline Approx 46 & 7213 & 7213 \\
\hline Approx 46.5 & & 3791 \\
\hline Approx 47 & 4527 & 4526 \\
\hline Approx 48 & 4205 & 4206 \\
\hline Approx 49 & 4050 & \\
\hline Approx 56 & & 3510 \\
\hline Approx 58 & & 3991 \\
\hline Approx 58.5 & 6793 & \\
\hline Approx 60 & & 4022 \\
\hline
\end{tabular}

${ }^{a}$ The "descriptor" molecules are components exclusive to the venom of a particular group (e.g., species group, species, or species gender). In general, "descriptor" molecules are components present in the majority of venom samples collected from a particular group and giving rise to a definitive mass spectral peak. However, in cases where only a single venom sample is obtained, the "descriptor" molecules are based solely on that single sample. Owing to the relationship of some of the Australian Funnel-web spiders, certain "descriptor" molecules are common to the specimens belonging to the species group, whereas other "descriptor" molecules are specific for a particular species variant or gender.

${ }^{b}$ Components common to the venom (i.e., possessing identical mass and retention time information $[M r \pm 3$ Daltons $]$ ) of both male and female specimens are highlighted in italics. 
Table 2

\section{Venom Mass Profile "Descriptor" Molecules Determined for Male and Female Specimens of Atrax sp.: Illawarra}

\begin{tabular}{ll}
\hline Elution time (min) & \multicolumn{1}{c}{ Components $(\mathrm{Mr}=\mathrm{Da})$} \\
\hline Approx 30 & 3944 \\
Approx 38 & 4472 \\
Approx 39 & 4579 \\
Approx 40 & 7600 \\
Approx 40.5 & 7105 \\
Approx 41.5 & 7443 \\
Approx 42.5 & 4850 \\
Approx 44 & 4102 \\
Approx 44.5 & 5666 (male only) \\
Approx 46 & 4221 \\
Approx 47 & 3820 (male only + juvenile male) \\
Approx 48 & 4519 (male only) \\
Approx 49 & 3948 \\
Approx 49.5 & 4911 (male only) \\
Approx 50 & 4181 (male only) \\
Approx 50.5 & 3851 \\
Approx 56 & 4410 (female only) \\
Approx 57 & 4278 \\
Approx 58 & 4361 (female only) \\
Approx 63 & 5355 \\
Approx 68 & 5338 \\
\hline
\end{tabular}

analysis (see Fig. 3) was only performed on the female and juvenile male venom samples owing to sample limitations of mature male venom.

The RP-HPLC/UV and RP-HPLC/ESI-MS analyses of the venom from Atrax sp.: Illawarra specimens illustrated that the composition of these venom samples is substantially different to the venom of the Atrax robustus: Sydney specimens. Furthermore, the venom of the juvenile male, mature male, and female Atrax sp.: Illawarra specimens is very similar in composition overall, but also contains a number of distinct differences.

The development of a venom mass profiling technique using RP-HPLC/UV and RP-HPLC/ESI-MS data, as illustrated above, has proven to be a useful tool for taxonomic distinction of different species variants of Australian Funnel-web spider belonging to the Atrax genus. The method also provides a method to distinguish between genders, and can distinguish between two very closely related specimens. 

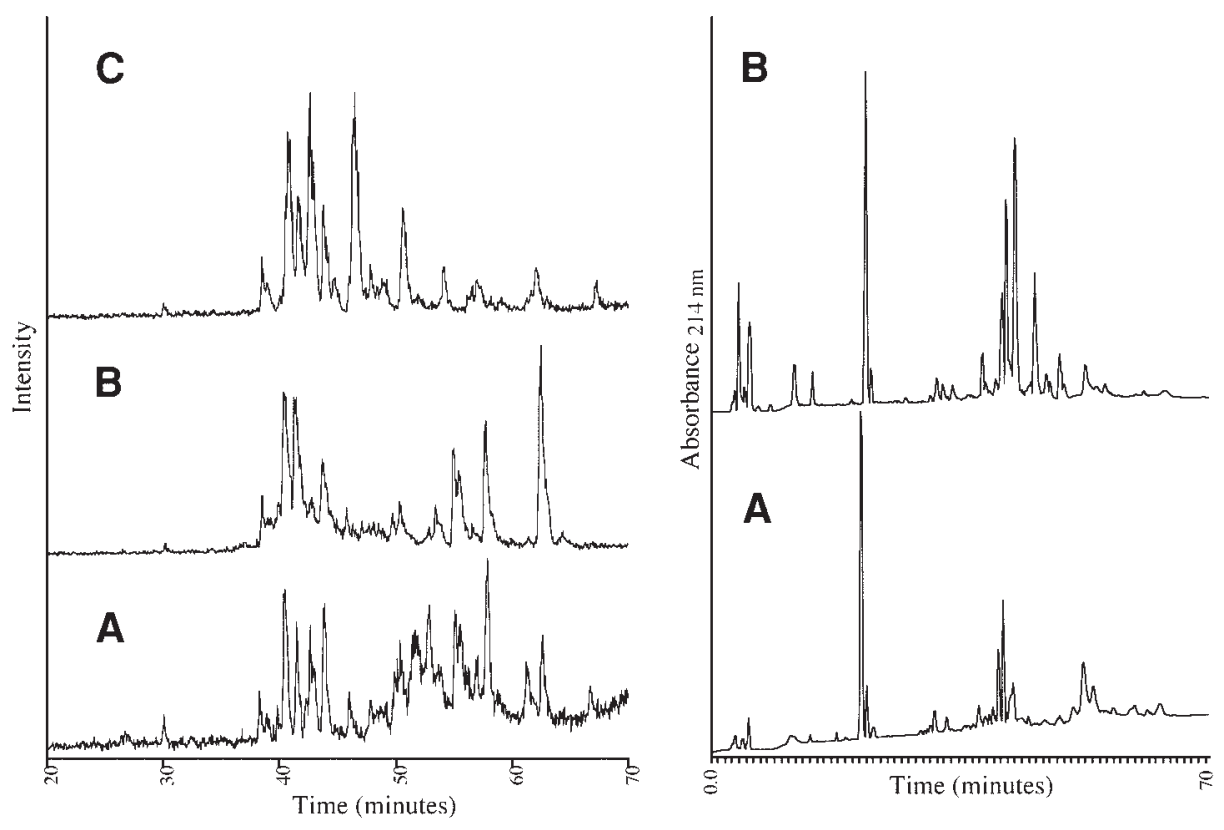

Fig. 3. A comparison of the RP-HPLC chromatograms from the juvenile, female, and mature male venom of Atrax sp.: Illawarra. (A) RP-HPLC/ESI-MS (left panel) and RP-HPLC/UV (right panel) chromatogram data obtained for the female Atrax sp.: Illawarra venom sample. (B) RP-HPLC/ESI-MS and RP-HPLC/UV chromatogram data obtained for the juvenile male Atrax sp.: Illawarra venom sample. (C) RPHPLC/ESI-MS chromatogram data for the venom sample obtained from the Atrax sp.: Illawarra postmaturing moult mature male specimen.

\section{Notes}

1. An important aspect in venom analysis is to minimize the loss of sample. This is even more important in cases where the study is performed on individual specimens, as a single specimen may only supply one venom collection before it dies, and some specimens may be very difficult to obtain (e.g., a particular species, or gender of a particular species or geographical variant). In these cases, as much information as possible has to be extracted from the solitary sample. Remember, once it's gone, it's gone for good!

2. MALDI-MS allows high-sensitivity analysis of large molecular weight compounds, including biomolecules such as proteins. MALDI involves ionization and transfer of a sample from a condensed phase to the gas phase by vaporization of a non-volatile solid matrix with laser radiation (18). This type of ionization is termed as "soft" ionization and results in molecule ionization without molecule fragmentation. The primary advantage of "soft" ionization is the ability to observe 
biomolecules in their native state. The principal disadvantage of MALDI-MS, for rapid venom profile analysis, is the requirement of matrix addition to the sample eliminating the possibility of on-line infusion RP-HPLC coupled mass spectrometry (RP-HPLC/MS). Venom profile analysis by MALDI-MS does not permit accurate mass/retention time correlation determination because of the necessity to acquire the RP-HPLC fractions first, followed by lyophilization and addition of the solid matrix of the fractions prior to mass spectrometry analysis. Furthermore, the reproducibility of MALDI-MS experiments on an individual sample suffers from variations in ionization of components. This variation results from inconsistent component crystallization with the matrix between crystals. Therefore, the mass spectrum obtained varies with respect to ion presence from crystal to crystal, and consequently the point of focus of the ionizing laser.

3. ESI-MS is also a "soft" ionization technique and involves the production of gaseous ionized molecules from a liquid solution (18). This is achieved by creating a fine spray of sample laced solvent in the presence of a strong electric field resulting in the formation of highly charged droplets. The charged droplets are electrostatically attracted to the inlet of the mass spectrometer where dry gas evaporates the solvent from the surface of the drops causing ions to leave the particle as the droplet size decreases. ESI results in the formation of multiply charged ions and, because the mass spectrometer measures the mass-to-charge ratio $(\mathrm{m} / \mathrm{z})$, allows the observation of relatively large molecules using a comparatively small mass range analyzer. Deconvolution of the multiply charged ion series can then be performed by a computer package and providing an accurate mass through averaging (18). The addition of a turbospray interface supplies heat and a greater dry gas flow to the solvent droplets and assists in the evaporation of the solvent. This increases the sensitivity of the mass spectrometer (up to $100 \times$ )*, particularly at higher solvent flow rates, and allows sufficient data collection from sample amounts down to a fraction of a single venom milking sample $(<10 \mu \mathrm{g}$ of crude venom). Because ESI-MS involves sample delivery in a solvent and a very "soft" ionization, on-line RP-HPLC coupled mass spectrometry of biomolecules can be performed (18). This ability to couple the RP-HPLC on-line with the mass spectrometer is the biggest advantage of RP-HPLC/ESI-MS over RP-HPLC/MALDITOF analysis of venom samples. RP-HPLC/ESI-MS allows direct flow of the eluting components to the mass spectrometer, in contrast to RP-HPLC/ MALDI-TOF, which requires separate sample collection, lyophilization, and matrix preparation prior to MS. The ability to incorporate on-line RP-HPLC into the analysis of complex mixtures, such as venom samples, greatly improves the sensitivity of detection by removing problems associated with the dynamic range of the detector. This problem results from ion saturation arising in analysis of complex samples possessing components of different concentrations. The maximum detection intensity resides with the most intense ion in the spectrum. A saturated

*Applied Biosystems homepage (http://www.appliedbiosystems.com/ab-mds_sciex/ products/ionsources/turboionspray.html). 
ion will, therefore, result in the suppression of weaker concentration or less ionizable components, suppressing the signal into the baseline noise. Separation of the components prior to mass spectral analysis (as in RP-HPLC/ESI-MS) reduces the effects of ion suppression as each component is analyzed individually. Despite the advantages of RP-HPLC/ESI-MS over RP-HPLC/UV, the UV data is still required, as the intensity of the mass spectrometer peaks depend on the ability of the component to ionize in the mass spectrometer and not the relative concentration of the component in the venom.

4. The venom collection container must be suitable for the creature in question (e.g., snakes require much larger venom collection containers than Funnel-web spiders do).

5. It is important when choosing a column for RP-HPLC/ESI-MS that the sample will not overload or underload the RP-HPLC column because this results in a loss of resolution. Furthermore, it is important to ensure that the amount of component eluting from the RP-HPLC column will not saturate the ESI-MS signal. This would lead to decreased sensitivity of low abundance molecules and decreased resolution in the RP-HPLC/ESI-MS chromatogram. Because detection by MS is far more sensitive than UV, the column choice and resultant flow rates are critical in the experimental setup. The experimental setup should always cater for the minimum amount of sample likely to be used, as this will always be the lower limit for detection (the detection of components eluting off RP-HPLC columns does not rely solely on the initial concentration of the sample added; the actual quantitative amount of sample and the solvent flow rate are important) and it is far easier to dilute concentrated samples than increase the concentration when only small amounts of sample are available. The experimental setup used should be kept as constant as possible to aid in the reproducibility of results and to also aid in the direct comparison of different samples.

6. Live or freshly dissected specimens are preferred because some venomous creatures can expel the venom from their venom glands upon freezing, and some specimens can be difficult to dissect frozen. Furthermore, dissection or freezing involves killing the specimen and limits the amount of venom that can be obtained from that specimen to a single collection. Venom extraction from dissected venom glands is also fraught with contamination problems (such as contaminating cellular byproducts and contents), providing a venom profile of the expelled venom that is not completely accurate. It has also been shown that the venom composition of Cone snail venom (19) and Funnel-web spider venom (20) differs between the venom gland and the expelled venom. "Milking" venom (collection of venom expelled by the creature from the venom duct) is the most preferred method, but can also have its problems with contamination. Very few venomous creatures are willing to expel venom in a manner such that it can be easily collected (snakes and the Australian Funnel-web spiders are the exception). Tricks have been developed to coax creatures such as Stonefish and Cone snails to be milked, however, the majority remain difficult. Electrical stimulation of the venom glands has become a popular method of "milking" venom and works by providing a pulsed electrical current to the area housing the venom apparatus $(\mathbf{1 6 , 2 1 )}$. This current 
causes rapid pulsed contraction of the muscles controlling venom output of the venom apparatus. In creatures where the venom apparatus is close to the mouth region (such as spiders) care must be taken to avoid contamination of the venom sample with regurgitated digestive tract material.

7. The use of polyethylene/polypropylene materials avoids problems associated with sample loss owing to the affinity of some venom molecules for glass. Although it is possible to avoid this problem by using silanized glassware, polyethylene/ polypropylene is preferred because its hydrophobic nature results in more complete sample transfer.

8. Lyophilization of the sample is preferably performed in something like a Savant speedvac to pellet crude venom. Standard driers without the added centrifugation step provided by the speedvac tend to result in lyophilized peptide up the sides of the tube, making reconstitution in small volumes difficult. Venom to be analyzed at a later date should be lyophilized and stored at $-20^{\circ} \mathrm{C}$, however, most highly disulfide bonded peptides are stable at room temperature for several days and even longer (lyophilized or in $30 \%$ organic solvent).

9. When reconstituting the sample to the desired analysis concentration, use a $30 \%$ solution of the organic solvent used in RP-HPLC. This concentration generally caters for all the hydrophilic and hydrophobic molecules, falling in the midrange of the extremes of typical component elution.

10. Where a group of molecules outside the range of interest are likely to interfere with those in the analysis range, the venom should be run through a size exclusion column (e.g., Microcon). However, this has the potential to alter the venom composition slightly through components binding to the column and should be avoided unless absolutely necessary.

11. The mass spectrometer signal must be very clean, with no contaminating flowthrough from the RP-HPLC columns, so the column must be washed thoroughly before use. Prior to commencing the RP-HPLC/ESI-MS analysis, it is a very good idea to attach the equilibrating RP-HPLC column to the mass spectrometer and equilibrate for a short period to ensure the column and mass spectrometer are clean. The mass spectrometer should also be calibrated prior to analysis. The narrow bore tubing used between the LC column, UV detector, and mass spectrometer should be suitable for the flow rate to be used (dependent on the column bore and packing) and kept as short as possible to ensure good correlation between retention times in the UV and MS data.

12. Contaminants dominating the signal intensity at either end of the mass range can be eliminated by reducing the mass scan range to just exclude the contaminating signal.

13. For RP-HPLC systems that routinely run at less than $200 \mu \mathrm{L} / \mathrm{min}$ with a small bore column (id $<2.1 \mathrm{~mm}$ ), a $5-\mu \mathrm{L}$ injection of a $1 \mathrm{mg} / \mathrm{mL}$ solution gives very good data, therefore, acceptable data can be safely obtained from loading $3-5 \mu \mathrm{L}$ of sample.

14. HPLC offers a couple of alternatives regarding the data that can be obtained. The UV detector can be removed if only MS data are required, however, it is typically 
desirable to incorporate the UV data. It is also possible to introduce a flow splitter in-line following the UV detector. This allows larger amounts of sample to be loaded and run at higher flow rates on larger bore columns. By splitting the flow so only a small portion goes to the mass spectrometer, the remainder can be directed for fraction collection providing the scientist with the UV data, mass spectrometer data, and fractionation in one step. The time frame for collection of all this data fits into the normal time frame taken to run the RP-HPLC/UV, vastly improving productivity. Furthermore, the homogeneity of the isolated fractions is known from the mass spectrometer data and can be used in further experiments such as reduction/alkylation leading to amino acid sequencing.

15. If a peak is evident in the RP-HPLC/UV chromatogram and not the RP-HPLC/ ESI-MS data, try scanning a wider mass range, or ionization in negative ion mode to obtain a mass.

\section{References}

1. Sutherland, S. K. and Tibballs, J. (2001) Australian Animal Toxins: The Creatures, their Toxins and Care of the Poisoned Patient. Second ed. Oxford University Press, South Melbourne, Australia.

2. Sheumack, D. D., Carroll, P. R., Hampson, F., et al. (1983) The isolation and Nterminal sequence of the lethal neurotoxin from the venom of the male Sydney Funnel-web spider. Atrax robustus. Toxicon. Suppl. 3, 397-400.

3. Collins, S. P., Comis, A., Tyler, M. I., Marshall, M., and Howden, M. E. H. (1995) Evidence for a high molecular weight pre-robustoxin molecule in the venom of the male Sydney funnel-web spider (Atrax robustus). Comp. Biochem. Physiol. 110C, 89-93.

4. Cain, T. C., Lubman, D. M., and Weber, W. J. J. (1994) Differentiation of bacteria using protein profiles from matrix-assisted laser desorption/ionization timeof-flight mass spectrometry. Rapid Commun. Mass Spectrom. 8, 1026-1030.

5. Chong, B. E., Wall, D. B., Lubman, D. M., and Flynn, S. J. (1997) Rapid profiling of $E$. coli proteins up to $500 \mathrm{kDa}$ from whole cell lysates using matrix-assisted laser desorption/ionization time-of- flight mass spectrometry. Rapid Commun. Mass Spectrom. 11, 1900-1908.

6. Krishnamurthy, T. and Ross, P. L. (1996) Rapid identification of bacteria by direct matrix-assisted laser desorption/ionization mass spectrometric analysis of whole cells. Rapid Commun. Mass Spectrom. 10, 1992-1996.

7. Holland, R. D., Wilkes, J. G., Rafii, F., et al. (1996) Rapid identification of intact whole bacteria based on spectral patterns using matrix-assisted laser desorption/ionization with time-of-flight mass spectrometry. Rapid Commun. Mass Spectrom. 10, 1227-1232.

8. Liang, X., Zheng, K., Qian, M. G., and Lubman, D. M. (1996) Determination of bacterial protein profiles by matrix-assisted laser desorption/ionization mass spectrometry with high-performance liquid chromatography. Rapid Commun. Mass Spectrom. 10, 1219-1226. 
9. Roda, A., Gioacchini, A. M., Seraglia, R., et al. (1997) A comparison of the analytical performance of sodium dodecyl sulfate-polycrylamide gel electrophoresis, electrospray and matrix-assisted laser desorption/ionization mass spectrometry in the study of the protein extract from Bothrops jararaca snake venom. Rapid Commun. Mass Spectrom. 11, 1297-1302.

10. Jones, A., Bingham, J. P., Gehrmann, J., et al. (1996) Isolation and characterization of conopeptides by high-performance liquid chromatography combined with mass spectrometry and tandem mass spectrometry. Rapid Commun. Mass Spectrom. 10, 138-143.

11. Bingham, J.-P., Jones, A., Lewis, R. J., Andrews, P. R., and Alewood, P. F. (1996) Conus venom peptides (conopeptides): Inter-species, intra-species and within individual variation revealed by ionspray mass spectrometry, in Biochemical Aspects of Marine Pharmacology (Lazarovici, P., Spiro, M., Zlotkin, E., eds.), Alaken Inc., www.alaken.com., Ft. Collins, CO, pp. 13-27.

12. Krishnamurthy, T., Prabhakaran, M., and Long, S. R. (1996) Mass spectrometric investigations on Conus peptides. Toxicon. 34, 1345-1359.

13. Romi-Lebrun, R., Martin-Eauclaire, M. F., Escoubas, P., et al. (1997) Characterization of four toxins from Buthus martensi scorpion venom, which act on apaminsensitive Ca2+-activated K+ channels. Eur. J. Biochem. 245, 457-464.

14. Escoubas, P., Celerier, M. L., and Nakajima, T. (1997) High-performance liquid chromatography matrix-assisted laser desorption/ionization time-of-flight mass spectrometry peptide fingerprinting of tarantula venoms in the genus Brachypelmachemotaxonomic and biochemical applications. Rapid Commun. Mass Spectrom. 11, 1891-1899.

15. Escoubas, P., Whiteley, B. J., Kristensen, C. P., Celerier, M. L., Corzo, G., and Nakajima, T. (1998) Multidimensional peptide fingerprinting by high performance liquid chromatography, capillary zone electrophoresis and matrix-assisted laser desorption/ionization time-of-flight mass spectrometry for the identification of tarantula venom samples. Rapid Commun. Mass Spectrom. 12, 1075-1084.

16. Escoubas, P., Chamot-Rooke, J., Stocklin, R., et al. (1999) A comparison of matrix-assisted laser desorption/ionization time-of-flight and liquid chromatography electrospray ionization mass spectrometry methods for the analysis of crude tarantula venoms in the Pterinochilus group. Rapid Commun. Mass Spectrom. 13, 1861-1868.

17. Sheumack, D. D., Baldo, B. A., Carroll, P. R., Hampson, F., Howden, M. E. H., and Skorulis, A. (1984) A comparitive study of properties and toxic constituents of funnel-web spider (Atrax) venoms. Comp. Biochem. Physiol. 78C, 55-68.

18. Siuzdak, G. (1996) Mass Spectrometry for Biotechnology. Academic, San Diego, CA.

19. Bingham, J. P. (1998) Novel Toxins from Conus: From Taxonomy to Toxins. University of Queensland, St. Lucia, Australia, p. 290.

20. Wilson, D. T. R. (2001) The Identification and Characterisation of Australian Funnel-web Spider Venom. University of Queensland, St. Lucia, Australia, p. 282. 
21. Atkinson, R. K. and Wright, L. G. (1990) A study of the necrotic actions of the venom of the Wolf Spider, Lycosa Godeffroyi, on mouse skin. Comp. Biochem. Physiol. 95C, 319-325. 